\title{
The Mathematical Structure of Superspace as a Consequence of Time Asymmetry
}

\author{
Mario Castagnino \\ Instituto de Astronomía y Física del Espacio. \\ Casilla de Correos 67, Sucursal 28. \\ 1428 BUENOS AIRES, Argentina.
}

July 11, 2021

\begin{abstract}
It is demonstrated how a convenient choice of the mathematical structure of the quantum cosmology superspace, precisely the definition of a convenient regular state superspace and the restriction of the dynamics to this space, yields directly to an irreversible evolution, in the classical (and semiclassical) phase of the universe, where:

- Decoherence and correlations take place and therefore give origin to a classical universe.

- The second law of thermodynamic is demonstrated.

- Connection with Reichenbach branch system idea can be implemented.

- Some rough coincidence with observational data are obtained.

- The arrows of time can be correlated.

- Time asymmetry can be explained as a state space asymmetry ( e. g. like a spontaneous symmetry breaking)

All these facts solve the problem of time-asymmetry and show that it is time asymmetry itself that defines the most important features of mathematical structure of superspace.

- e-mail: Castagni (a) iafe.uba.ar

- Pacs Nrs. 05.20-y, 03.65 BZ, 05.30-d.
\end{abstract}




\section{Introduction}

The role of physics is to explain nature in the best possible way. Therefore physicists use to consider a set of physical phenomena and to choose the best axiomatic structure to mimic these phenomena. This axiomatic structure contains a mathematical structure and a set of axioms (or postulates, or principles, or hypotheses), stated using the language of the chosen mathematical structure. If the mathematical structure is the most naturally related to the set of phenomena and a minimal number of axioms is used, physicist say that they have explained nature in the best possible way. But, frequently a better mathematical structure and a smaller set of axioms are found to explain a larger set of physical phenomena, then physicists say that they understand the problem even better because, in fact, they have found a better explanation $i$. e. a more economical one. But the chosen mathematical structure and the chosen axioms cannot be explained by themselves, since the only motivation of the choice is to mimic nature in the best possible way.

Thus, physical phenomena are not a consequence of the chosen mathematical structure, quite on the contrary, the choice of the mathematical structure is a consequence of the physical phenomena that we are trying to explain.

Gravitation was explained by Einstein choosing a riemannian manifold as mathematical structure and postulating that particles space-time paths were the geodesic of such a manifold, etc. The only explanation of this choice is that the theoretical motions, so described, mimic real motions better than the motions described using other curves or other kind of manifolds (flat space-time, projective manifolds, etc.). But the riemannian manifold and the geodesics cannot be explained by themselves. In fact, the choice of the riemannian manifold, as the mathematical structure to explain gravity, is really motivated by the method we use to measure time and distance [1].

It also happens that, when a new or unfamiliar mathematical structure is introduced, some physicists think that the new structure is introduced by hand, because they do not realize that every mathematical structure was introduced by hand in, order to explain nature in the best possible way.

In the perspective of this pedagogical (perhaps pedantic but necessary) introduction we will choose: 


\subsection{The set of phenomena.}

The set of phenomena considered in this paper will be those of usual quantum cosmology (QC) based in Wheeler-De Witt (WDW) equation [2], [3], plus those of irreversible statistical quantum physics, like the definitions of the various arrows of time, the final equilibrium state, decoherence, correlations, etc. (see the book [4] where almost all subjects, results, and problems can be found).

\subsection{The mathematical structure.}

$\mathrm{QC}$ is based in WDW equation;

$$
H \Psi=0
$$

where $H$ is the hamiltonian operator of the model for the considered universe and $\Psi$ the wave function of the universe (that will be also called $|\Psi\rangle$ ) [3], [5]. Usually $H$ is well defined by the model we are studying, but the real mathematical nature of $\Psi$ is not so well defined. In fact, let us call " $q$ " the configuration variables and $Q$ the configuration space, then $\Psi=\Psi(q)$ is a function of the configuration variables. We must define the space of wave functions $\Psi$. Let us suppose that this space has a discrete basis $\mathcal{P}$ of certain functions of the $q$ (let us say polynomials of the $q$ or polynomials multiplied by convenient dumping factors for those $q, s$ that goes to infinity). But, this set is not enough because usually wave functions belong to a complete space. Then we must complete the space expanded by $\mathcal{P}$ with some topology. The usual idea is to complete the space expanded by $\mathcal{P}$ with the topology of the norm. Even if the choice of a norm contains a very important physical problem: the definition of probability, we are now exclusively interested in the mathematical problem of how to complete $\mathcal{P}$ with an adequate topology. Then we have really two problems:

i.- We must define a norm in the space expanded by $\mathcal{P}$ something like [6]:

$$
\|\Psi\|=\int_{Q} \Psi^{*}(q) \Psi(q) d q
$$

But we can also foliate $Q$ with hypersurfaces $\Sigma$ and define [2]:

$$
\|\Psi\|=\int_{\Sigma} \Psi^{*}(q) \partial_{n} \Psi(q) d \sigma_{n}
$$


where $n$ is the normal to $\Sigma$ and $d \sigma_{n}$ is the hypervolume of the hypersurface $\Sigma$ differential element (this quantity will be positive definite in a convenient, subspace of superspace). Or we can consider that really $\Psi$ is an operator and then we must go to a third quantization [7], etc. So we have many possibilities to define the norm. Actually we are trying to copy usual quantum mechanics and the essential property to define an adequate norm is that it would be a constant under time evolution. But in QC there is no time [8]. [9] [10]. In fact, eq. (1) is not a Schroedinger equation but an eigen equation that defines a "stationary" eigenfunction, with no time variable in it. Therefore, as the definition of norm is intimately intertwined with the problem of the definition of time in $\mathrm{QC}$, and this problem is not solved [8], [11], [12] it is not possible to give a definitive definition of norm. Really in this paper we will adopt the conservative attitude that this problem cannot be solved and therefore $\mathrm{QC}$ is a timeless theory [8], [9]. As a consequence it is very difficult to define a satisfactory norm.

ii.-But even if a norm would be chosen, say (2), we have another problem. If we complete the space expanded by $\mathcal{P}$ with (2) we will find $\mathcal{H}=L^{2}(Q)$ the Hilbert space of square integrable functions over $Q$. But $H$ is an operator with derivatives and the functions of $\mathcal{H}$ are, in general, not derivable, they are just square integrable, so for an arbitrary function $\Psi$ of $\mathcal{H}, H \Psi$ has no meaning (and we would have the same problem with the other norms). So we must choose another topology to complete the space expanded by $\mathcal{P}$. Let us consider the Schwarz class functions, namely functions that can be derived an infinite number of times and that are well behaved in the eventual infinite of coordinates $q$ (precisely they vanish faster than any polynomial). The set of Schwarz functions is a nuclear space. Then if we complete the space expanded by $\mathcal{P}$ with the corresponding topology, which is not a norm topology but a nuclear one [13], we obtain a space $\mathcal{S}$ where we can derive any number of times. If $\mathcal{S}^{\times}$is the space of (anti)linear functional over $\mathcal{S}$, our mathematical structure is really the Gel'fand triplet (or rigged Hilbert space):

$$
\mathcal{S} \subset \mathcal{H} \subset \mathcal{S}^{\times}
$$

Then we can say that $\mathcal{S}$ is the superspace of regular states where we can do all our computations and $\mathcal{S}^{\times}$is the superspace of generalized states, where we will find usual distributions like Dirac's deltas or plane (or curved) waves, that can be used to expand regular states. In ordinary quantum mechanics 
$\mathcal{H}$ would be the superspace of states, but in $\mathrm{QC}$ this space looses almost all its importance because we do not know what norm we have to use for sure (even if, to compute probabilities, we will use norm (2) in spite of the fact that we have other possible choices[14]), while $\mathcal{S}$ and $\times$ are well defined, since their definitions are norm-independent. The "inner product" $\langle F \mid \Psi\rangle$ of an element of $\mathcal{S}^{\times}$written as a "bra", $<F \mid$, with an element of $\mathcal{S}$ written like a "ket", $\mid \Psi>$, is also well defined, since it is just the functional acting over a regular state, (also the product in the inverted order is well defined, if we set $<\Psi|F>=<F| \Psi>^{*}$ ). So $\mathcal{S}$ is the arena where timeless QC works: where time and norm have disappeared from the mathematical structure and are substituted by the nuclear topology of $\mathcal{S}$.

But if we use $\mathcal{S}$ as a regular space we cannot encompass (in the most economical way) irreversible statistical quantum mechanics, which has an asymmetry that it is not contained in $\mathcal{S}$. There are only two causes for asymmetry in nature: either the laws of nature are asymmetric or the solutions of the equation of the theory are asymmetric. E. g.: The laws of nature are asymmetric in the case of the weak interaction. The solutions of the theory are asymmetric in the case of spontaneous symmetry breaking.

Time asymmetry is not an exception. Thus, if we want to retain the time-symmetric laws of nature (namely the symmetry of eq. (四)) the only way we have to explain the time-asymmetry of the universe or its subsystems is to postulate that the space of solutions is not time-symmetric, namely we use the second cause of asymmetry. So the proper way to solve the problem is simply to define a realistic time-asymmetric space of physical admissible solutions $\phi_{-}$, i. e. an adequate mathematical structure for superspace. $\phi_{-}$ will contain the states that evolve in an admissible way (e.g.: Gibbs ink drop spreading in a glass of water, a sugar lump solving in a cup of coffee, etc.) and will not contain the non admissible evolutions ( the ink or the sugar concentrating spontaneously and creating the drop or the lump). The problem is to choose $\phi_{-}$in the best possible way. As we will see we will choose one with the required asymmetry. We will follow a heuristic approach: we will suppose that $H$ is endowed with all the properties necessary to define a reasonable universe. Obviously these "realistic hamiltonians" are the only $H$ that we must consider. Therefore practically, in $H$ there must be always some fields, like the mater field, the electromagnetical field, and also the gravitational field (namely only the graviton field). So we would write this 
hamiltonian in a midi-superspace way as:

$$
H=h_{n f}\left(g_{j}, \pi j\right)+h_{f}\left(\varphi, p_{\varphi}\right)+h_{i}\left(g_{i j}, \varphi\right)
$$

where $h_{n f}\left(g_{j}, \pi_{j}\right)$ is the "no field" hamiltonian (a function of a discrete set of modes of the gravitational field $g_{j}$ and the corresponding momenta $\left.\pi_{j}\right), h_{f}$ is the "fields" hamiltonian, (let us say the continuous set of all the modes of just one field $\varphi$, that represents all the physical fields in our model, and the corresponding momenta $\left.p_{\varphi}\right)$, and $h_{i}$ is the interaction hamiltonian among fields and no fields (usually an interaction among only the configuration variables).

To complete the panorama, (using the Gel'fand-Maurin theorem [16]) we can find, for the partial hamiltonian $h_{n f}+h_{f}$, a spectral decomposition:

$$
h_{n f}+h_{f}=\sum_{i} \omega_{i}|i><i|+\int_{0}^{\infty} \omega|\omega><\omega| d \omega
$$

where the first term of the r.h.s. corresponds to the spectral decomposition of $h_{n f}$, (cf. eq. 5) and therefore to a discrete spectrum, and the second one to the spectral decomposition of $h_{f}$ and therefore to a continuous spectrum. Some negative eigenvalues " $\omega$ " must appear because $H$ is not bounded from below. Any state $\mid \Psi>\in \mathcal{S}$ can be expanded as:

$$
\left|\Psi>=\sum_{i}\right| i><i\left|\Psi>+\int_{0}^{\infty}\right| \omega><\omega \mid \Psi>d \omega
$$

This is the spectral decomposition of $\mid \Psi>$ in basis $\{|i>,| \omega\rangle\}$. In order that $\mid \Psi>$ would satisfy the WDW eq. (1) it must be:

$$
H\left|\Psi>=\sum_{i} \omega_{i}\right| i><i\left|\Psi>+\int_{0}^{\infty} \omega\right| \omega><\omega\left|\Psi>d \omega+h_{i}\right| \Psi>=0
$$

Most likely this equation can be solved.

Also, in every reasonable model, the universe will end in a classical phase, going first through semiclassical one. Namely, a variable $a$ will exist (one of the $g_{j}$ or a function of the $g_{j}$ ) such that a time $\eta$ can be defined as a function of $a$. When $\eta \rightarrow \infty$ we will obtain a classical geometry $g_{\mu \nu}^{\text {out }}$ for the universe. It will be the most probable geometry of the universe (i. e. the geometry that appears most frequently) [17]. Using time $\eta$ we can transform eq. (11) in a Schroedinger equation, with the corresponding 
hamiltonian $h$ [5]. Then using $h$ and the classical geometry $g_{\mu \nu}^{\text {out }}$ we can find a semi-classical vacuum state $\mid 0$, out $>$ for the fields (the so called adiabatic vacuum), that diagonalizes the hamiltonian $h=h\left(g_{\mu \nu}^{\text {out }}\right)=h$ (out) (computed in the geometry $\left.g_{\mu v}^{\text {out }}\right)$, the creation and annihilation operators related to this vacuum, and the corresponding Fock space. But the only essential ingredient we need to implement the theory is the $h($ out $)$. Then, using these objects, we can find a set of eigenvectors $\mid \omega$, out $>$ [18], such that:

$$
h \mid \omega, \text { out }>=\omega \mid \omega, \text { out }>
$$

where $\omega$ is a continuous eigenvalue of $h$ (say $0 \leq \omega<\infty$ ) 21]. So:

$$
h=\int_{0}^{\infty} \omega \mid \omega, \text { out }><\omega, \text { ou } \mid d \omega
$$

where $\mid \omega$, out $>\in \mathcal{S}^{\times}$, thus if $\mid \Psi>\in \mathcal{S}, \quad\langle\omega$, out $| \Psi>$ is well defined and so $h \mid \Psi>$. The existence of this kind of expansions can be also considered a consequence of Gel'fand-Maurin theorem [16]. But all these manipulations are just formal, so we will be sure that what we are doing is correct only in concrete examples, as the one in the next section. In fact, we will find all this mathematical elements in the model presented there. Surely we will also find these elements in more complex models [19.

Now, let us define our new regular state space $\phi_{-} \subset \mathcal{S}$. Precisely, we can promote $\omega$ to a complex variable $z$ and ask, not only that $\mid \Psi>\in \mathcal{S}$, but also that $\langle z$, out $\mid \Psi\rangle$ would be an analytic function in the lower complex halfplane (precisely that $\langle\omega$, out $| \Psi>\in H_{-}^{2}$ being $H_{-}^{2}$ the Hardy functions class from below). These functions $\mid \Psi>$ belong to a space $\phi_{-}$such that:

$$
\phi_{-} \subset \mathcal{S}
$$

and we have a new Gel'fand triplet:

$$
\phi_{-} \subset \mathcal{H} \subset \phi_{-}^{\times}
$$

then we know that [13]:

$$
\mathcal{S}^{\times} \subset \phi_{-}^{\times}
$$

So we have restricted the regular state superspace and simultaneously we have enlarged the generalized state superspace so we will have more general spectral expansions (this fact will be of outmost importance). But, as we 
can as well choose the upper complex halfplane (precisely $<\omega$, out $\mid \Psi>\in H_{+}^{2}$ being $H_{+}^{2}$ the Hardy functions class from above), we also have another space $\phi_{+}$such that:

$$
\phi_{+} \subset \mathcal{S}
$$

and another Gel'fand triplet:

$$
\phi_{+} \subset \mathcal{H} \subset \phi_{+}^{\times}
$$

We also know that 13:

$$
\mathcal{S}^{\times} \subset \phi_{+}^{\times}
$$

Now we can also say that we have obtained the space $\phi_{-}$completing the space expanded by $\mathcal{P}$ with the nuclear topology of $\phi_{-}$, namely the $\mathcal{S}$-topology restricted to $\phi_{-}$(and the same thing can be said about $\phi_{+}$). Clearly this topology is endowed with a new asymmetry which $\mathcal{S}$ does not have. Precisely, this asymmetry allows us to choose between $\phi_{-}$or $\phi_{+}$even if we maintain all the symmetries of $H$ [20]. Thus we can break one of these symmetries, restricting the dynamics to superspace $\phi_{-}$which then would be considered as the superspace of regular states. As we will see this restriction produces the desired time asymmetry. Frequently physicists make analytic continuation in the complex energy plane supposing that some functions are analytic in one halfplane only. In these cases they are implicitly using the kind of mathematical structure we have explicitly introduced here, so the idea is, by no means, new.

Thus our mathematical structure will essentially be (12), $\phi_{-}$will be our superspace of regular states, where we must find the states that satisfy the WDW eq. (11) and $\phi_{-}^{\times}$will be our generalized state superspace. From (11) we see that we have restricted our regular state superspace, so nothing unphysical can happen. We are just adding a new requirement to regular states, in order to assure their asymmetry. Furthermore $\phi_{-}$is dense in $\mathcal{H}$, so if someone would believe that the real "physical" states belong to $\mathcal{H}$ we could argue that these "physical" states can be approximated, by regular states of $\phi_{-}$, as close as we want.

\subsection{The axiomatic structure.}

We do not pretend to give a completely rigorous axiomatic structure in this paper (but just an approximation of it). Furthermore we do not know if the 
proposed axiomatic structure is unique. We are just proposing a first draft of a complete axiomatic structure, so we will call to our axioms just hypotheses.

As we would like that the equilibrium state would be contained in our theory we must also consider mixed states $\rho$ and, therefore, the spaces:

$$
\Phi_{-}=\phi_{-} \otimes \phi_{-}, \ldots \mathcal{L}=\mathcal{H} \otimes \mathcal{H}, \ldots \Phi_{-}^{\times}=\phi_{-}^{\times} \otimes \phi_{-}^{\times}
$$

and work in the Liouville triplet:

$$
\Phi_{-} \subset \mathcal{L} \subset \Phi_{-}^{\times}
$$

where $\mathcal{L}$ is the usual Liouville space of ordinary mixed states, which actually we will never use, since our regular state superspace is $\Phi_{-}$. So, let $\rho$ be a selfadjoint density matrix. Then our main hypotheses are:

$H_{1}$. The state $\rho$ of the universe satisfies the equations [15]:

$$
H \rho=0
$$

$H_{2}$. The state $\rho$ of the universe belongs to the superspace $\Phi_{-}$, i. e.:

$$
\rho \in \Phi_{-}
$$

$H_{3} . \rho\left(q, q^{\prime}\right)$ is proportional to the correlation between the configurations $q$ and $q^{\prime}$ and $\rho(q, q)$ is proportional to the probability to find the configuration $q$ in the universe.

These three axioms correspond to the three elements necessary to go into QC mentioned in the introduction of paper [3]: dynamics, "initial condition" (precisely definition of the physically admissible states of the universe in a timeless formalism), and interpretation. Of course $\mathrm{H}_{2}$ alone does not fix the actual state of the universe, but any state of the universe we choose to build our theory must be contained in $\Phi_{-}$, if we want that time-asymmetry would appear in a natural way. In this paper we do not address the problem to find the real and unique state of the universe, but only to define a superspace of admissible states such that the universe would turns out to be time-asymmetric.

We will see how far we can go with this axiomatic structure. The paper is organized as follows: 
- In section 2 we introduce our model, its semiclassical approximation and we obtain a new spectral decomposition, using the regular superspace of hypothesis $\mathrm{H}_{2}$.

- In section 3 we obtain the evolution equation of the states.

- In section 4 up to section 9 we find the physical characteristics of the model.

- In section 10 we draw our main conclusions.

- Two appendices, containing further explanations and the way to fix the two \pm signs of the theory, complete the paper.

\section{The Model.}

Let us see how we can implement all we have said in a simple model.

Let us consider the model of section 3, of ref. [22], or better the one of ref. [23], where a Robertson-Walker metric:

$$
d s^{2}=a^{2}(\eta)\left(d \eta^{2}-d x^{2}-d y^{2}-d z^{2}\right)
$$

is studied, (we will mostly consider the flat space geometry case). The total action is: $S=S_{g}+S_{f}+S_{i}$, being $S_{g}\left(=S_{n f}\right)$ the gravitational action, $S_{f}$ the usual action of an spinless massless field $\varphi$, conformally $\left(\xi=\frac{1}{6}\right)$ coupled , and $S_{i}$ the interaction given by a mass term in Robertson-Walker geometry. The gravitational action is given by:

$$
S_{g}=M^{2} \int d \eta\left[-\frac{1}{2} \stackrel{\bullet}{a}^{2}-V(a)\right]
$$

where $M$ is the Planck's mass, $\eta$ is the conformal time, $a$ is the RobertsonWalker scale , $\dot{a}=\frac{d a}{d \eta}$, and $V(a)$ is a potential that arises from the spatial curvature, a possible cosmological constant, and, eventually a classical matter

field. $V(a)$ is a potential with a bounded support contained in $0 \leq a \leq a_{1}$, with $a_{1} \gg 0$, (in many examples $V(a)$ is a function of $a^{2}$ and $V(a)$ strongly vanishes, for $a^{2} \rightarrow \infty$, 23] so our potential can be considered as a good approximation of these examples). This case is the simplest of all, but we 
believe that the main features, that we will find, will also be present in more general cases.

The WDW eq. (11) for our model is:

$$
H \Psi(a, \varphi)=\left(h_{g}+h_{f}+h_{i}\right) \Psi(a, \varphi)=0
$$

where (in our the flat space geometry case):

$$
\begin{gathered}
h_{g}=\frac{1}{2 M^{2}} \partial_{a}^{2}+M^{2} V(a) \\
h_{f}=-\frac{1}{2} \int_{k}\left(\partial_{\varphi_{k}}^{2}-k^{2} \varphi_{k}^{2}\right) d \mathbf{k} \\
h_{i}=\frac{m^{2} a^{2}}{2} \int_{k} \varphi_{k}^{2} d \mathbf{k}
\end{gathered}
$$

where $m$ is the mass of the scalar field, and $k^{2}=|\mathbf{k}|^{2}$, where $\frac{\mathbf{k}}{a}$ is the linear momentum of the field, in the flat case we are working (in the two other cases, namely open and close space geometry, the integrals of eqs. (25) and (26) are integrations on adapted coordinates, in the open case, and summatories, in the close case, where $\mathbf{k}$ is substituted by a discrete variable; see the corresponding equations in 22]).

Now, let us go to the semiclassical case using the WKB method [3], [5], [24]. So let:

$$
\Psi(a, \varphi)=\exp [i M S(a)] \chi(a, \varphi)
$$

To satisfy WDW eq. (11), at order $M^{2}$, the principal Jacobi function $S(a)$ must satisfy the Hamilton-Jacobi equation:

$$
\left(\frac{d S}{d a}\right)^{2}=2 V(a)
$$

Now we can define the time, in our up to now, timeless theory. It is the (semi)classical time parameter $\eta=\eta(a)$ given by:

$$
\frac{d}{d \eta}=\frac{d S}{d a} \frac{d}{d a}= \pm \sqrt{2 V(a)} \frac{d}{d a}
$$

From eqs. (28) and (29) we can find the set of classical solutions:

$$
a= \pm f(\eta, C)
$$


where $C$ is an arbitrary integration constant. Using different values for this constant and different choices of the \pm sign we obtain different classical geometries (in more general cases many constants would be necessary). For $a>a_{1}$, it is $\sqrt{2 V(a)}=0$ (since $V(a)$ have a bounded support, contained in $\left[0, a_{1}\right]$ ), and we cannot define time $\eta$ using eq. (29), thus we must choose another hand for our clock to define the time there. To avoid this problem let us consider that when $a>a_{1}$ it is $\sqrt{2 V(a)}=\varepsilon=$ const. $\cong 0$. We can always make $\varepsilon=0$ to reobtain. the real case. Then $a$ will be

$$
a= \pm \varepsilon \eta+C
$$

So we can see that the potential can also be considered as a function with bounded support in variable $\eta$. We will consider that $\varepsilon>0$. The role of $C$ is just to fix the origin of time, so we can take any $C$ we want . As the coupling is conformal we will have well defined vacua [25], 26]. In particular we can consider two scales $a_{\text {in }}$ and $a_{\text {out }}$ such that $0<a_{\text {in }} \ll a_{1}$, $a_{\text {out }} \gg a_{1}$ and define the $\mid 0$, in $>, \mid 0$, out $>$ vacua there. (We can as well transform all the equations to the non-rescaled case, consider the proper time $t=\int a d \eta$, and the physical momentum $\frac{\mathbf{k}}{a}$ and define the $\mid 0$, out $>$ in the $\eta \rightarrow \infty$ limit, as in the appendix A of paper [23], but here we will use the first simpler formalism.)

In this model we have:

$$
h(a)=h_{f}\left(\varphi_{k}\right)+h_{i}\left(a, \varphi_{k}\right)
$$

where we have omitted the $\varphi_{k}$ in $h(a)$. Then:

$$
h(a)=\frac{1}{2} \int_{k}\left[-\frac{\partial^{2}}{\partial \varphi_{k}^{2}}+\Omega_{k}^{2}(a) \varphi_{k}^{2}\right] d \mathbf{k}
$$

where (cf. eqs. (25) and (26)):

$$
\Omega_{k}^{2}(a)=m^{2} a^{2}+k^{2}
$$

So $h(a)$ is a time dependent hamiltonian, where all its time dependence comes from a scale variable mass $m^{2} a^{2}$. It is well known (25], 26]) that we can diagonalize this time dependent hamiltonian at $a_{\text {in }}$ and at $a_{\text {out }}$ and define the corresponding vacua, the corresponding creation and annihilation operator, 
and the corresponding Fock spaces. For the out-geometry the vacuum will be the adiabatic vacuum, since $a_{\text {out }} \gg a_{1}$, therefore all the out elements will coincide with those defined in the introduction. In fact, the out-geometry is almost constant during the final time evolution (that goes up to $\eta \rightarrow \infty$ ) and therefore they correspond to the geometry with the maximum probability. $h\left(a_{\text {out }}\right)$ reads:

$$
h\left(a_{\text {out }}\right)=\int_{k} \Omega_{k}\left(a_{\text {out }}\right) a_{\text {out }, k}^{\dagger} a_{\text {out }, k} d \mathbf{k}
$$

where $a_{\text {out }, k}^{\dagger}$ and $a_{\text {out }, k}$ are the creation and annihilation operator corresponding to the out vacuum. With these objects we can construct the Fock space with a basis:

$$
\left|k_{1} . k_{2}, \ldots k_{n, \text { out }}>=\right|\{k\}, \text { out }>\sim a_{\text {out }, k_{1}}^{\dagger} a_{\text {out }, k_{2}}^{\dagger} \ldots a_{\text {out }, k_{n}}^{\dagger} \mid 0, \text { out }>
$$

where we have called $\{k\}$ the set $k_{1}, k_{2}, \ldots k_{n}$. These states are eigenvectors of $h\left(a_{\text {out }}\right)$, precisely;

$$
h\left(a_{\text {out }}\right) \mid\{k\}, \text { out }>=\omega\left(a_{\text {out }}\right) \mid\{k\}, \text { out }>
$$

(of course, this equation corresponds to eq. (9) ) where:

$$
\omega\left(a_{\text {out }}\right)=\sum_{k \in\{k\}} \Omega_{k}(\text { out })
$$

We can use this energy to label the eigenvectors as:

$$
\mid\{k\}, \text { out }>=\mid \omega,[k], \text { out }>
$$

where $[k]$ is the remaining set of labels necessary to define the vector unambiguously. $\{\mid \omega,[k]$, out $>\}$ is an orthonormal basis, namely:

$$
\begin{gathered}
<\omega,[k], \text { out } \mid \omega^{\prime}\left[k^{\prime}\right], \text { out }>=\delta\left(\omega-\omega^{\prime}\right) \delta\left([k]-\left[k^{\prime}\right]\right) \\
1=\int_{0}^{\infty} d \omega \int_{[k]} \mid \omega,[k], \text { out }><\omega,[k], \text { out } \mid d[k]
\end{gathered}
$$

where the meaning of the symbols related with $[k]$ is evident. In the close space geometry case the indices would be discrete and the integral a summatory. 
All the same can also be done at $a_{i n}$. We can now define the S-matrix between the in and out states:

$$
S_{\omega,[k] ; \omega^{\prime},\left[k^{\prime}\right]}=<\omega,[k], \text { in } \mid \omega^{\prime},\left[k^{\prime}\right], \text { out }>
$$

According to papers [27], [28], this matrix has an infinite set of complex poles as we will demonstrate in section 4 (also an example is given in 23] and using this paper and [26] and [29] other examples can be obtained).

If we forget the indices $[k]$ and consider again the eq. (9), we see that $\mid \omega,[k]$, out $>$ is the $\mid \omega$, out $>$ of this equation. In the introduction we have defined the triplets (12) and (15) only using the hamiltonian $h($ out $)=h\left(a_{\text {out }}\right)$. These triplets correspond to a Fock space defined for $a_{\text {out }}$. But there will also be two similar triplets defined in the Fock space at $a_{i n}$. We make the following choice (motivated on reasons that will be apparent in a moment): for the in-Fock space we will use functions $\mid \varphi>\in \phi_{+i n}$, namely such that $\mid \varphi>\in \mathcal{S}$ and $\langle\omega$, in $\mid \varphi\rangle \in H_{+}^{2}$, and for the out-Fock space we will use functions such that $\mid \varphi>\in \phi_{-, \text {out }}$, namely such that $\mid \varphi>\in \mathcal{S}$ and $\langle\omega$, out $| \varphi>\in H_{-}^{2}$. So the $\phi_{-}$of the introduction is now $\phi_{-, \text {out }}$ and our regular states belong to this space [30]. The role of $\phi_{+, \text {in }}$ is to allow us to define the corresponding functional space $\phi_{+, i n}^{\times}$so we can use the functionals of this space in some spectral decompositions [31]. As both vacua, at $a_{\text {in }}$, and $a_{\text {out }}$ are well defined and the particle production between these vacua is finite and the theory is implementable [32]. We can then multiply the state of both Fock spaces.

So let us again write eq. (41) with no $[k]$ :

$$
1=\int_{0}^{\infty} d \omega \mid \omega, \text { out }><\omega, \text { out } \mid
$$

Of course there is an analogous equation for the "in" case. Now using this equation and eq. (42) we have:

$$
\mid \omega, \text { out }>=\int_{0}^{\infty} d \omega^{\prime} \mid \omega^{\prime}, \text { in }><\omega^{\prime} . \text { in } \mid \omega, \text { out }>=\int_{0}^{\infty} d \omega^{\prime} \mid \omega^{\prime}, \text { in }>S_{\omega^{\prime} . \omega}
$$

Then:

$$
1=\int_{0}^{\infty} d \omega \int_{0}^{\infty} d \omega \mid \omega^{\prime}, \text { in }>S_{\omega^{\prime} \cdot \omega}<\omega, \text { out } \mid
$$

or:

$$
<\psi\left|\varphi>=\int_{0}^{\infty} d \omega \int_{0}^{\infty} d \omega^{\prime}<\psi\right| \omega^{\prime}, \text { in }>S_{\omega^{\prime}, \omega}<\omega, \text { out } \mid \varphi>
$$


Now let $\mid \varphi>\in \phi_{-, \text {out }}$ and $|\psi\rangle \in \phi_{+, \text {in }}$. Then $\langle z$, out $| \varphi>\in H_{-}^{2},<z$, in $|\psi\rangle \in$ $H_{+}^{2}$ and therefore $\langle\psi| z$, in $>\in H_{-}^{2}$. So in the integrand of the last equation all the factors are analytic in the lower halfplane, with the exception of $S_{\omega, \omega^{\prime}}$, which has an infinite number of poles $\Omega_{n}$ as we have already said. Then we can choose any curve $\Gamma$, beginning at the origin, and going below all the poles of the lower halfplane up to the infinity of the positive real axis [33]. We can now change the integration contour of eq. (46) from $[0, \infty)$ to the curve $\Gamma$. If we add the pole contribution as in papers [34], [35], and [36], we obtain:

$$
\begin{aligned}
<\psi \mid \varphi> & \left.=\sum_{n}<\psi \mid n+>\langle n-\mid \varphi\rangle+\int_{\Gamma} d z \int_{\Gamma} d z^{\prime}\langle\psi| z^{\prime}, \text { in }\right\rangle S_{z^{\prime}, z}<z, \text { out }|\varphi\rangle= \\
& =\sum_{n}<\psi \mid n+>\langle n-\mid \varphi\rangle+\int_{\Gamma} d z\langle\psi| z+>\langle z-\mid \varphi\rangle
\end{aligned}
$$

where the summatory comes from the residues of the poles (each pole $\Omega_{n}$ is labelled by a discrete index $n$ of course $\left.\operatorname{Im} \Omega_{n} \leq 0\right)$ and:

$$
|z,->=| z, \text { out }>, \ldots \ldots\left|z+>=\int_{\Gamma} d z^{\prime} S_{z^{\prime} z}\right| z^{\prime}, \text { in }>
$$

Then, in a weak sense, we have found a new spectral decomposition of 1 :

$$
1=\sum_{n}|n+><n-|+\int_{\Gamma} d z|z+><z-|
$$

Following the same procedure with $\langle\psi| h($ out $)|\varphi\rangle$ we can obtain the spectral decomposition of $h($ out) (always in a weak sense):

$$
h(\text { out })=\sum_{n} \Omega_{n}|n+><n-|+\int_{\Gamma} z|z+><z-| d z
$$

We have three possibilities to choose the curve $\Gamma$ :

i.- To use all possible curves $\Gamma$ as in paper [36].

ii.- To take the curve $(-\infty, 0]$, in the second sheet, as in ref. [35], provided we have a good behavior at infinity of the lower halfplane.

iii.- To use the Nakanishi trick [37, as in paper [39], namely to define tilded functionals such that:

$$
\int_{\Gamma}|z+><z-| d z=\int_{0}^{\infty}|\widetilde{\omega+}><\widetilde{\omega-}| d \omega
$$


We will use this last notation. Then we have:

$$
\begin{gathered}
1=\sum_{n}|n+><n-|+\int_{0}^{\infty} d \omega|\widetilde{\omega+}><\widetilde{\omega-}| \\
h(\text { out })=\sum_{n} \Omega_{n}|n+><n-|+\int_{0}^{\infty} \omega|\widetilde{\omega+}><\widetilde{\omega-}| d \omega
\end{gathered}
$$

(see also [38] and paper [34], where the Nakanishi trick is explain). From its own definition it is evident that $|n->| z-,>, \mid \widetilde{\omega-}>\in \phi_{-, \text {out }}^{\times}$, since these vectors are functionals over $\mid \varphi>\epsilon \phi_{-, \text {out }}$ and that $|n+>| z+,>, \mid \widetilde{\omega+}>\epsilon$ $\phi_{+, i n}^{\times}$since these vectors are functionals over $\mid \psi>\in \phi_{+, i n}$. Restoring the $[k]$ and eliminating the tilde (as we will always do below) the last equation reads:

$$
h(\text { out })=\sum_{n} \Omega_{n}|n+><n-|+\int_{0}^{\infty} d \omega \int_{k} \Omega_{k}|\omega,[k]+><\omega,[k]-| d[k]
$$

but we will continue with the previous shorthand notation and we will not write the $[k]$ any more. It can be proved that the bases $\{|n+>,| \omega+>$ \},$\{|n->,| \omega->\}$ are a biorthonormal system, [35], [36], and [39] namely:

$$
\begin{aligned}
& <n-\left|n^{\prime}+>=\delta_{n n^{\prime}}, \ldots \ldots \ldots \ldots<n-\right| \omega+>=0 \\
& <\omega-|n+>=0, \ldots<\omega-| \omega^{\prime}+>=\delta\left(\omega-\omega^{\prime}\right)
\end{aligned}
$$

From all these equation we have that:

$$
\begin{gathered}
h(\text { out })\left|n+>=\Omega_{n}\right| n+> \\
<n-\mid h(\text { out })=\Omega_{n}<n-\mid
\end{gathered}
$$

where $\Omega_{n}$ is a complex eigen value, and $\mid n+>$ are right eigenvectors, and $<n-\mid$ left eigenvector of $h($ out $)$. Even if $h($ out $)$ is hermitian, it has complex eigenvalues because we are using a new spectral decomposition, which is only possible because we are working in a convenient Gel'fand triplet. This fact will be the main tool that we will use below. The eigenvalues and their squared will be written as:

$$
\begin{gathered}
\Omega_{n}=\omega_{n}-\frac{i}{2} \gamma_{n}, \ldots . . \gamma_{n} \geq 0 \\
\Omega_{n}^{2}=m^{2} a(\text { out })^{2}+z_{n}
\end{gathered}
$$

compared with eq. (34) this equation shows that there are also poles in the variable $k^{2}$ which are complex numbers. 


\section{Time evolution.}

Coming back to the WKB expansion (27), if we now consider the next order and the time defined in eq. (29), the function $\chi(a, \varphi)$ must satisfy the Schroedinger equation:

$$
i \frac{d \chi}{d \eta}=h(\eta) \chi
$$

where $h(\eta)$ is hamiltonian $h$ written as a function of $\eta$. Even if this hamiltonian is time depending we can consider that for scales $a>a_{\text {out }}$ there is not particles creation and therefore we have an invariant adiabatic vacuum $\mid 0$, out $>$ and a definitive pole structure for the S-matrix [23], so for $a(\eta)>a_{\text {out }}$ expansion (54) will always have the same structure. Thus the time evolution of $\chi$ will be:

$$
\chi(\eta)=\exp \left[-i \int h(\eta) d \eta\right] \chi(0)
$$

From this equation we can obtain some conclusions:

i.- In particular the time evolution of the right eigenvector $\mid n+>$ reads:

$$
\left|n(\eta)+>=\exp \left[-i \int \Omega_{n}(\eta) d \eta\right]\right| n(0)+>
$$

because even if the pole structure remains fixed the poles move as can be seen in the example of ref. [23], eq. (3.3). So, from eq. (57) we can see that, if there are some $\gamma_{n}>0$, some of these eigenvectors have a dumped evolution. Therefore, these eigenvectors correspond to decaying states. Thus our formalism naturally yields decaying states that vanish towards, the direction of time, that we can call the future.

ii.- Using eq. (52) we can expand any function $\mid \varphi>\in \phi$ _as:

$$
\left|\varphi>=\sum_{n}\right| n+><n-\left|\varphi>+\int_{0}^{\infty}\right| \omega+><\omega-\mid \varphi>d \omega
$$

then its time evolution will be:

$$
\begin{gathered}
\left|\varphi(\eta)>=\sum_{n} \exp \left[-i \int \Omega_{n}(\eta) d \eta\right]\right| n+><n-|\varphi\rangle+ \\
+\int_{0}^{\infty} \exp \left[-i \int \Omega_{k}(\eta) d \eta\right]|\omega+><\omega-| \varphi>d \omega
\end{gathered}
$$


where all terms in the summatory, such that $\gamma_{n} \neq 0$, have a decaying evolution, while the rest of the terms and the integral have an oscillatory behavior. So in the time evolution of (almost) any state we have a decaying term that vanishes towards the future.

iii.- In this way the asymmetry introduced in hypothesis $\mathrm{H}_{2}$ produces an effective time asymmetry, because it allows to define a future time direction. Moreover, it can be proved that, if in eq. (60) the evolution operator is considered as an operator from space $\phi_{-}$to space $\phi_{-}$, namely if we restrict the dynamics to space $\phi_{-}$, eq. (60) is only defined for $\eta \geq 0$. Therefore the evolution operator cannot be inverted and so, it is really an irreversible operator (see [35], [34], 40]).

iv.- Let us consider the case of mixed states. For a mixed state $\rho \in \Phi_{-}$ we can generalize the spectral decomposition (62) to obtain:

$$
\begin{gathered}
\rho=\sum_{n, m} \rho_{n m}|n+><n+|+\sum_{n} \int_{0}^{\infty} \rho_{n \omega}|n+><\omega+| d \omega+ \\
+\int_{0}^{\infty} \sum_{n} \rho_{\omega n}|\omega+><n+| d \omega+\int_{0}^{\infty} \int_{0}^{\infty} \rho_{\omega \omega^{\prime}}|\omega+><\omega+| d \omega d \omega^{\prime}
\end{gathered}
$$

Repeating the computation of the pure state case, we can compute the time evolution of state $\rho(\eta)$. Since either $\gamma_{n}=0$ or $\gamma_{n}>0$ there will be oscillating terms and dumped ones then we obtain:

$$
\rho(\eta)=\rho_{*}(\eta)+\exp \left[-\frac{1}{2} \int \gamma d \eta\right] \rho_{1}(\eta)
$$

where the first term, of the r.h.s. is an oscillatory term and the second a decaying term, where we have written a first factor corresponding to the slowest dumping factor, namely $\gamma$ is the smallest of the non-zero $\gamma_{n}$. When $\eta \rightarrow \infty$ we have:

$$
\rho(\eta) \rightarrow \rho_{*}(\eta)
$$

$\rho_{*}(\eta)$ is a thermodynamical equilibrium state. In fact, since in its evolution there are not dumping factors, it behaves like an ordinary stable quantum state and its entropy is time-constant, namely the one that corresponds to thermodynamical equilibrium (below we will normalize this constant to zero). It is logically a non stationary oscillatory equilibrium state, because, even if it is in thermic equilibrium, the field cannot go to dynamical equilibrium since, in our simple model, there are no interaction terms among 
the field components. If these terms would be present, new dumping factors would also be present and the final equilibrium would be a stationary state [41].

This is the essence of our formalism. Below we will see the results that we can obtain if we follow this road.

\section{Decoherence and correlations.}

In reference [23], using our formalism, it is proved that, if the S-matrix has an infinite number of complex poles, we have decoherence and that, in unstable states, configuration and momentum are correlated, in such a way that the universe ends in a classical phase. In this demonstration hypothesis $\mathrm{H}_{3}$ plays an essential role. In paper 23] it was not proved that, in general, the Smatrix, relevant for our problem, has an infinite set of complex poles, but that set was computed in one example, while other examples were proposed.

Here we will not repeat the demonstration of ref. [23], but we will complete this paper observing that using a potential, with a bounded support, as in the present paper, the existence of an infinite set of poles is a consequence of ref. 27] and [28].

In fact: A massive scalar field, conformally coupled, in metric (21) satisfies Klein-Gordon equation:

$$
\left(\nabla_{\mu} \nabla^{\mu}+m^{2}+\frac{1}{6} R\right) \psi=0, \ldots . . R=6 a^{-3} \frac{\partial^{2} a}{\partial \eta^{2}}
$$

This equation leads, by variable separation to:

$$
\psi=\frac{1}{(2 \pi)^{\frac{3}{2}} a(\eta)} f(\eta) \exp ( \pm i k x)
$$

and $f(\eta)$ satisfies a generalized oscillator equation with time-depending frequency:

$$
f^{\prime \prime}+\omega^{2}(\eta) f=0, \ldots \omega=\left[a^{2}(\eta) m^{2}+k^{2}\right]^{\frac{1}{2}}
$$

On the other hand, in ordinary quantum mechanics, the stationary solution $u(x)$ of a massive particle in a potential $W(x)$ satisfies:

$$
u "(x)+\omega^{2}(x) u(x)=0, \ldots \ldots \omega(x)=[2 m(E-W(x))]^{\frac{1}{2}}
$$


So both phenomena can be mathematically related according to the analogy:

$$
a^{2}(\eta) m^{2} \leftrightarrow-2 m W(x), \ldots . . k^{2} \leftrightarrow 2 m E
$$

More details about this analogy can be obtained from paper [27].

Now from reference [28], page. 218, we know that the S-matrix of a cut-off potential $W(x)$, namely a potential with a bounded support, has an infinite number of complex poles. Our potential is $\sim a^{2}(\eta)$ which is practically a constant for $a \gg a_{1}$, since $\varepsilon \cong 0$, and exactly a constant if we consider the real value $\varepsilon=0$ so, subtracting this constant final value, we can say that it is a cut-off potential, with a S-matrix endowed with an infinite set of poles.

Almost all potentials used in the literature of Quantum Field theory in Curved Space-time [26] are very well behaved in the infinities and can be mimicked by this, bounded support, kind of potentials (this is not the case for some QC potentials, that we will discuss in the conclusions). So the existence of an infinite set of poles seems quite a general feature of the theory. Thus, using the equation of paper [23], it can be proved that our formalism leads to decoherence, to correlations, and to the outcome of a classical universe, in a natural way.

Finally in particular subsystems of the universe the S-matrix has poles if unstable quantum states exist in the subsystem [35]. Of course, these poles will also appear in any complete S-matrix of the universe.

\section{Entropy}

Let $\rho(\eta)$ be the density matrix, of the universe or one of its subsystems, for a physical admissible state $\left(\rho \in \Phi_{-}\right)$and let $\rho_{*}(\eta)$ be the corresponding thermodynamical equilibrium matrix. In the universe these matrices are related by eq. (65). The hamiltonian of the subsystem is necessarily a term of the general hamiltonian of eq. (23) and its S-matrix must have poles if the subsystem is not trivial (see papers [34] and [35]). So we can repeat all what we have said for the universe for the case of the subsystem and we can also choose a t-asymmetric regular space state for the subsystem. We must take care that the dumping or future direction of the subsystem coincides with the dumping or future direction of the universe for consistency. I. e. the local and global arrows of time must coincide. Then we will also find eq. (65) for the subsystem. The only difference would be that, if the subsystem 
hamiltonian $h$ is no time dependent (with respect e. g. to the proper time $t$ ) in the integral of eq. (65) must be subtituted by the usual product $h t$.

Then we can define the conditional entropy $S=S\left[\rho(\eta) \mid \rho_{*}(\eta)\right]$, of states $\rho(\eta)$ with respect to state $\rho_{*}(\eta)$ [42 both for the universe or the subsystem:

$$
S=S\left[\rho(\eta) \mid \rho_{*}(\eta)\right]=-\operatorname{tr}\left\{\rho(\eta) \log \left[\rho_{*}^{-1}(\eta) \rho(\eta)\right]\right\}
$$

such that $S\left[\rho_{*}(\eta) \mid \rho_{*}(\eta)\right]=0$, namely the entropy vanishes at equilibrium. We can, as well, use the corresponding classical definition, since really we are interested in the classical phase of the universe, but, in order to use only one notation, we will use the quantum formulae.

From eq. (65) we see that:

$$
\operatorname{tr} \rho(\eta)=\operatorname{tr} \rho_{*}(\eta)=1 \Rightarrow \operatorname{tr} \rho_{1}(\eta)=0
$$

i.e.: if the states $\rho(\eta)$ and $\rho_{*}(\eta)$ are normalized as it should be, $\rho_{1}(\eta)$ has a vanishing trace, thus $\rho_{1}(\eta)$ is not a state but the coefficient of a correction of the equilibrium state to obtain state $\rho(\eta)$. The vanishing of the trace of $\rho_{1}$ is directly proved in papers [34, [40], 43], using our formalism. Now if we expand the logarithm in eq. (72), and use eqs. (73) we obtain:

$$
S=S\left[\rho(\eta) \mid \rho_{*}(\eta)\right]=-\exp \left[-\frac{1}{2} \int \gamma d \eta\right] \operatorname{tr}\left[\rho_{*}^{-1}(\eta) \rho_{1}^{2}(\eta)\right]+\ldots
$$

where the dots symbolize higher order terms [44].

This entropy has the property:

$$
\lim _{\eta \rightarrow \infty} S\left[\rho(\eta) \mid \rho_{*}(\eta)\right]=0
$$

namely the entropy evolves towards its null equilibrium value. This is so because the prefactor in eq. (74) dominates any other time variation, since $\rho_{*}(\eta)$ is usually oscillatory (namely it will be oscillatory in the case a, but not in the case b, see below) and $\rho_{1}(\eta)$ has oscillatory terms and dumping factors that vanish faster than the dominant decaying factor.

In eq. (72) we have two matrices: $\rho(\eta)$ and $\rho_{*}(\eta)$, then we have also two possibilities. Either both matrixes have the same kind of evolution or they have different ones. The first is the general case, but the second case appears when $\rho_{*}(\eta)$ follows a different evolution due to, e.g., an external agency. Let us consider the two cases: 
a.- Both matrices follow the same evolution law. Namely, if we have a time variable hamiltonian $h(\eta)$, as in the case of the universe, the evolution will be:

$$
\rho(\eta)=\exp \left[-i \int_{\eta^{\prime}}^{\eta} l(\eta) d \eta\right] \rho\left(\eta^{\prime}\right), \ldots \ldots . . \rho_{*}(\eta)=\exp \left[-i \int_{\eta^{\prime}}^{\eta} l(\eta) d \eta\right] \rho_{*}\left(\eta^{\prime}\right)
$$

where $l(\eta)$ is the corresponding Liouville operator, i. e., $l(\eta) \rho=h(\eta) \rho-$ $\rho h(\eta)$.

In subsystems of the universe where the hamiltonian $h$ is not a propertime variable( e. g. in subsystem which does not expand or contract due to an external agency), we would have:

$$
\left.\rho(t)=\exp \left[-i l\left(t-t^{\prime}\right)\right] \rho\left(t^{\prime}\right) \ldots \ldots \ldots \ldots \rho_{*}(t)=\exp \left[-i l\left(t-t^{\prime}\right)\right)\right] \rho_{*}\left(t^{\prime}\right)
$$

where $l$ is the Liouville operator corresponding to hamiltonian $h$ and $t$ is the proper time. In this case the equilibrium matrix $\rho_{*}(t)$ also evolves in the same way than matrix $\rho(t)$. Now, from what we have said in Sec.3, point iii, eq. (76) is only valid if $\eta>\eta^{\prime}$. Analogously, if the subsystem S-matrix has poles and we have also chosen for the subsystem a similar admissible function space $\Phi_{-}$, eq. (77) is only valid if $t-t^{\prime} \geq 0$ or $t \geq t^{\prime}$. Therefore the last two evolutions are irreversible. Now, from ref. [42] (using the classical-quantum analogy since we are in the classical phase, the "exp " operator of the last two equations will be a Frobenius-Perron operator, and we can use the classical definition of conditional entropy) we know that:

$$
S\left[\rho(\eta) \mid \rho_{*}(\eta)\right] \geq S\left[\rho\left(\eta^{\prime}\right) \mid \rho_{*}\left(\eta^{\prime}\right)\right], \ldots S\left[\rho(t) \mid \rho_{*}(t)\right] \geq S\left[\rho\left(t^{\prime}\right) \mid \rho_{*}\left(t^{\prime}\right)\right]
$$

respectively. It would be $=$ if the evolution operators would be reversible, but they are not (consider also eq.( 74)). Then these entropies are really monotonically growing. Therefore we have proved the second law of thermodynamics, for the whole universe or for any non trivial subsystem. So our formalism yields this fundamental law naturally (compare with the much more complicated coarse graining method of paper [45]).

The demonstration is based in the fact that (in both cases) $\rho$ is an admissible state (like the ink drop spreading in the glass of water), so $\rho \in \Phi_{-}$and $\gamma_{n} \geq 0$. If we would have taken $\rho \in \Phi_{+}$it would be $\gamma_{n} \leq 0$ and the entropy would decrease showing that:

i.- In the case of the subsystem. $\Phi_{+}$is the space of non admissible solution (the ink drop contracting spontaneously). In fact, in this case the arrow of 
time is the one of the universe and not the one of the subsystem, and in the subsystem we will see a decaying of the entropy, showing that the state is not physically admissible.

ii.- In the case of the universe. Going from $\Phi_{-}$to $\Phi_{+}$we have simply changed our convention (since all possible arrows of time are embodied in the universe), calling the "future" the direction of decreasing entropy.

b.- Let us now consider the important case of a subsystem of the universe: the matter and radiation within an expanding universe ( i. e. we do not take into account the entropy of the gravitational field, and we consider that this filed as an external agency that expands the space, where the matter and the radiation are located). Then the conditional entropy is not necessarily monotonically increasing, at least for short times. In fact, we cannot use eq. (78) since $\rho_{*}(\eta)$ does not satisfy an equation like $\left(76_{2}\right)$, because its evolution is fixed by the external universe expansion. This is completely logical since $S\left[\rho(\eta) \mid \rho_{*}(\eta)\right]$ is just the matter radiation entropy (with no gravitational field entropy contribution) in an expanding (or contracting) universe with a equilibrium state $\rho_{*}(\eta)$, that varies in an independent way and it is well known that matter (let us say a gas) can have decreasing entropy into a variable geometry (let us say a box with moving walls). A phenomenological study of the problem can be found in ref. [46], 47]. In this case what we have called up to now $S$ is just the entropy gap $\Delta S$ with respect to a variable maximal possible entropy $S_{\max }$. The actual entropy, which grows monotonically, is $S=S_{a c t}=S_{\max }+\Delta S$. But $\Delta S$ has not this property. Furthermore, the diminishing of $\Delta S$ for short times is welcome, as we will see in the next two sections.

Finally let us consider the origin of the "miracle" that allows us to define a growing entropy with no coarse-graining. The miracle is produced by the generalized spectral decompositions (62) or (64) which yield the evolution (63) with only dumping (and oscillatory) factors, which, in turn, are only possible if we introduce an asymmetry, like the one of hypothesis $\mathrm{H}_{2}$. Namely if we restrict the regular state space in a t-asymmetric way we obtain also the asymmetric generalized states of expansions and eqs. (62) and (64). Is this fact so strange? Experimentally we know that we only have a finite set of physical measurements to fix a quantum state, e. g. we know the value of the wave function in a finite set of point of the configuration space $Q$. In the limit we will have a set of discrete data that allows us to define by interpolation e. g. a polynomial belonging to the set of polynomials $\mathcal{P}$ of 
the introduction. But in order to work we need the whole wave function complete superspace and therefore we must complete the space expanded by the $\mathcal{P}$. In the ordinary theory we complete the space expanded by the $\mathcal{P}$ with the topology of the norm of $\mathcal{H}$ or the nuclear one of $\mathcal{S}$. Up to this point we have a time-symmetric theory. If we want to introduce time asymmetry we coarse-grain the system, to obtain a new complete space of relevant states. In the new theory we complete using Hardy-Schwartz function (i. e. we complete with the nuclear topology of $\phi_{-}$). So we only use one step, instead of two, and obtain the same physical results. But, really both methods have essentially the same physical base: we have only a finite (or discrete) amount of information and this information must be worked out, somehow, to obtain the complete space of regular states of the theory. So, as expected, there is no miracle.

\section{The entropy gap.}

In this section we study the universe entropy gap $\Delta S=S_{a c t}-S_{\max }$, following a qualitative idea of Paul Davies [46]. We will complete this idea actually computing the entropy gap after decoupling time. Therefore we change our model, it will be still homogeneous and isotropic, with metric ( 21), but obviously the particle production is finished, so we will consider that we are simply in a flat geometry, matter dominated, universe.

It is well known that the isotropic and homogeneous expansion of the universe is a reversible process with constant entropy 48]. In this case the matter and the radiation of the universe are in a thermic equilibrium state $\rho_{*}(t)$ at any time $t$. As the radiation is the only important component, from the thermodynamical point of view, we can choose $\rho_{*}(t)$ as a black-body radiation state [49], i. e. $\rho_{*}(t)$ will be a diagonal matrix with main diagonal:

$$
\rho_{*}(\omega)=Z T^{-3} \frac{1}{e^{\frac{\omega}{T}}-1}
$$

where $T$ is the temperature, $\omega$ the energy, and $Z$ a normalization constant (50], eqs. (60.4) and (60.10)). The total entropy is:

$$
S=\frac{16}{3} \sigma V T^{3}
$$


([50], eq. (60.13)) where $\sigma$ is the Stefan-Boltzmann constant and $V$ a commoving volume.

Let us consider our isotropic and homogeneous model of universe with scale $a$. Any commoving volume evolves as $V \sim a^{3}$, and, since from the conservation of the energy-momentum tensor and radiation state equation, we know that $T \sim a^{-1}$, we can verify that $S=$ const. Thus the irreversible nature of the universe evolution is not produced by the universe expansion, even if $\rho_{*}(t)$ has a slow time variation.

Therefore, after decoupling time, the main process that has an irreversible nature is the burning of unstable $H$ in the stars (which produces $H e$ and, after a chain of nuclear reactions, $F e$ ). This unstable state produces poles in the corresponding S-matrix and a nuclear reaction process, with mean life-time $t_{N R}=\gamma^{-1}$. Therefore, using eq. (65), and considering that $\gamma$ is constant (under proper time variation), since it corresponds to a local process considered in the paragraph "a" of the last section, (or simply on phenomenological grounds) we can then say that the state of the universe, at time $t$, is:

$$
\rho(t)=\rho_{*}(t)+\rho_{1} e^{-\gamma t}+0\left[e^{-\gamma t}\right]
$$

where $\rho_{1}$ is a certain phenomenological coefficient, which is constant in time since all the time variation of nuclear reactions is embodied in the exponential law $e^{-\gamma t}$. Also on phenomenological grounds, we can foresee that $\rho_{1}$ must peak strongly around $\omega_{1}$, the characteristic energy of the nuclear process. All these reasonable phenomenological facts can also be theoretically explained in different ways, e. g.: Eq. (81) can be computed with the theory of paper [36]. In reference [40] it is explicitly proved that $\rho_{1}$ peaks strongly at the energy $\omega_{1}$. So using eq.(72) we can compute the entropy gap:

$$
\Delta S=-\operatorname{tr}\left[\rho \log \left(\rho_{*}^{-1} \rho\right)\right]
$$

Using now eq. (81), and considering only times $t \gg t_{N R}=\gamma^{-1}$ we can expand the logarithm, as in eq. (74), to obtain:

$$
\Delta S \approx-e^{-\gamma t} \operatorname{tr}\left(\rho_{*}^{-1} \rho_{1}^{2}\right)
$$

where we have used eq. (73). We now introduce the equilibrium state (79) for $\omega \gg T$. Then:

$$
\Delta S \approx-Z^{-1} T^{3} e^{-\gamma t} \operatorname{tr}\left(e^{\frac{\omega}{T}} \rho_{1}^{2}\right)
$$


where $e^{\frac{\omega}{T}}$ is a diagonal matrix with this function as diagonal. But as $\rho_{1}$ is peaked around $\omega_{1}$ we arrive to a final formula for the entropy gap:

$$
\Delta S \approx-C T^{3} e^{-\gamma t} e^{\frac{\omega_{1}}{T}}
$$

where $C$ is a positive constant.

Let us now compute the time evolution of the entropy gap. We have computed $\Delta S$ for times larger than decoupling time and therefore, as $a \sim t^{\frac{2}{3}}$ and $T \sim a^{-1}$, we have:

$$
T=T_{0}\left(\frac{t_{0}}{t}\right)^{\frac{2}{3}}
$$

where $t_{0}$ is the age of the universe and $T_{0}$ the present temperature. Then:

$$
\Delta S \approx-C_{1} e^{-\gamma t} t^{-2} e^{\frac{\omega_{1}}{T_{0}}\left(\frac{t_{0}}{t}\right)^{\frac{2}{3}}}
$$

where $C_{1}$ is a positive constant. Drawing the corresponding curve [47] it can be seen that $\Delta S$ has a maximum at $t=t_{c r_{1}}$ and a minimum at $t=t_{c r_{2}}$. Let us compute these critical times. The time derivative of the entropy reads:

$$
\dot{\Delta} S \approx\left[-\gamma-2 t^{-1}+\frac{2}{3} \frac{\omega_{1}}{t_{0} T_{0}}\left(\frac{t_{0}}{t}\right)^{\frac{1}{3}}\right] \Delta S
$$

This equation shows two antagonic effects. The universe expansion effect is embodied in the second and third terms in the square brackets, being an external agency to the matter-radiation system such that, if we neglect the second term, it tries to increase the entropy gap and, therefore, to take the system away from equilibrium (as we will see the second term is practically negligible). On the other hand, the nuclear reactions embodied in the $\gamma$ term, try to convey the matter-radiation system towards equilibrium. These effects become equal at the critical times $t_{c r}$ such that:

$$
\gamma t_{0}+2 \frac{t_{0}}{t_{c r}}=\frac{2}{3} \frac{\omega_{1}}{T_{0}}\left(\frac{t_{0}}{t_{c r}}\right)^{\frac{1}{3}}
$$

For almost any reasonable numerical values this equation has two positive roots: $t_{c r_{1}} \ll t_{0} \ll t_{c r_{2}}$. Precisely: 
i.- For the first root we can neglect the $\gamma t_{0}$-term and we obtain:

$$
t_{c r_{1}} \approx t_{0}\left(3 \frac{T o}{\omega_{1}}\right)^{\frac{3}{2}}
$$

(this quantity, with minus sign, gives the third unphysical root).

ii.- For the second root we can neglect the $2\left(t_{0} / t_{c r}\right)$-term, and we find:

$$
t_{c r_{2}} \approx t_{0}\left(\frac{2}{3} \frac{\omega_{1}}{T_{0}} \frac{t_{N R}}{t_{0}}\right)^{3}
$$

Let us make now some numerical estimates. We must choose numerical values for four parameters: $\omega_{1}=T_{N R}, t_{N R}=\gamma^{-1}, t_{0}$, and $T_{0}$.

$T_{N R}$ and $t_{N R}$ can be chosen between the following values [51]:

$$
\begin{gathered}
T_{N R}=10^{6} . . \text { to.. } 10^{8} .{ }^{0} \mathrm{~K} \\
t_{N R}=10^{6} \text {..to. } .10^{9} \text {.years }
\end{gathered}
$$

while for $t_{0}$ and $T_{0}$ we can take:

$$
\begin{gathered}
t_{0}=1.5 \times 10^{10} . \text { years } \\
T_{0}=3^{0} \mathrm{~K}
\end{gathered}
$$

In order to obtain a reasonable result we choose the lower bounds for $T_{N R}$ and $t_{N R}$ and for $t_{c r_{1}}$ we obtain :

$$
t_{c r_{1}} \approx 1.5 \times 10^{3} \text {.years }
$$

So $t_{c r_{1}}$ is smaller than the decoupling time and it should not be considered since the physical processes before this time are different than those we have used in our model. Also, we must only consider times $t>t_{N R}=\gamma^{-1}$, in order to use eq. (83).

For $t_{c r_{2}}$ we obtain:

$$
t_{c r_{2}} \preceq 10^{4} t_{0}
$$

From eqs. (94) and (95) we can see that really $t_{c r_{1}} \ll t_{0} \ll t_{c r_{2}}$. Thus:

-From $t_{N R}$ to $t_{c r_{2}}$ the expansion of the universe produces a decreasing of entropy gap, according to Paul Davies prediction [46]. Also, it probably produces a growing order, and therefore the creation of structures like clusters, galaxies and stars [52]. 
-After $t_{c r_{2}}$ we have a growing of entropy, a decreasing order and a spreading of the structures: stars energy is spread in the universe, which ends in a thermic equilibrium [53]. In fact, when $t \rightarrow \infty$ the entropy gap vanishes (se eq. (87)) and the universe reaches a thermic equilibrium final state.

$t_{c r_{2}} \preceq 10^{4} t_{0}$ is the frontier between the two periods. Is the order of magnitude of $t_{c r_{2}}$ a realistic one? In fact it is, since $10^{4} t_{0} \approx 1.5 \times 10^{14}$ years after the big-bang all the stars will exhaust their fuel [53], so the border between the two periods should have this order of magnitude. Further, it should also be smaller than this number. This is precisely the result of our calculations (see also letter [47]).

So we are at the edge of a correct physical prediction, even if our model is extremely naive and simplified: an homogeneous universe and besides we have neglected the higher order terms in eq. (81) which perhaps may be important for finite times. Besides in the real universe nuclear reactions take place within the stars, that can only be properly considered in a inhomogeneous geometry. Nevertheless, this rough numerical estimate shows the consistency of all the theory. Furthermore the decreasing of the entropy gap, in the period $t_{N R}<t<t_{c r_{2}}$, will be crucial in the next section.

\section{The branch system.}

The set of irreversible processes within the universe, each one beginning in an unstable non-equilibrium, state can be considered a branch system [46, [54], . Namely, every one of these processes begin in a non-equilibrium state, such that, this state was produced by a previous process of the set. E. g.: Gibbs ink drop (initial unstable state) spreading in a glass of water ( irreversible process) is only probable (since the probability to create an ink drop by fluctuations is extremely small) if there was first an ink factory, which extracted the necessary energy from an oven, where coal (initial unstable state) was burnt (branched irreversible process); in turn coal was created with energy coming from the sun, where $H$ (initial unstable state) is burnt into $\mathrm{He}$ (branched irreversible process); finally $H$ was created using energy obtained from the unstable initial state of the universe (the absolute initial state of the branch system). Therefore, using this hierarchical chain, all the irreversible processes are related to the cosmological initial condition, the only one that must be explained. Let us observe that: 
i.- The branch system defines its own arrow of time the branch arrow of time (BAT), as the direction that goes from the unstable initial state of every member of the system towards equilibrium. Probably the BAT is the most useful of all arrows of time, since it is present in any irreversible local process.

ii.- Once we have the branch system the irreversible evolution of each system is easy to explaining, since once we have understood the origin of the initial unstable state of each irreversible process within the universe (even if we have not yet discussed the origin of the initial state of the whole universe) it is not difficult to obtain Lyapunov variables (or irreversible evolution equations), if we consider, e. g. that the subsystems where these processes take place are not isolated. If it is so, forces of stochastic nature penetrate from the exterior of each subsystem and, it is well known, that if we add stochastic terms to time-symmetric evolution equation, we obtain time-asymmetric ones, yielding Lyapunov variables e. g. a non-decreasing entropy [42]. We can also consider that each subsystem has an enormous amount of information and we are able to measure, compute, and control a part of this information, that we will call relevant. If we neglect the rest of the information, the irrelevant one, we can obtain also irreversible evolution equations and Lyapunov variables [42], [55]. These two procedures can be considered within the coarse-graining usual formalism.

iii.-But of course, if we follow the ideas of this paper, we will use more refined mathematical tools, and in each of the subsystem, introduce a model similar to the one we have used for the whole universe, as we have done in section 5, introducing the hypothesis $\mathrm{H}_{2}$ in each subsystem. (It has already been done in papers [34], 35], [40], and the same results are obtained, i. e. irreversible evolution equation, Lyapunov variables, etc.) Then we see that entropy grows in each subsystem provided the state of the subsystem would be chosen among the physically admissible states of space $\Phi_{-}$. Then each subsystem of the branch system begins in an unstable, low entropy, state and evolves towards thermal equilibrium. The physical non-admissible states (those of space $\Phi_{+}$) correspond to theoretical evolutions that would only exist before the instant of creation of the subsystem (the instant when we put the ink in the glass of water ) evolving with decreasing entropy, towards that instant (namely the ink drop contracting spontaneously). These evolutions simply do not exist in nature because, before the instant of its creation, the subsystem really does not exist as such. Before that instant a different 
subsystem exits with different evolution laws (the ink factory that creates the ink drop). Therefore all the scenario turns out to be realistic and satisfactory.

iv.- So only one problem is left: Why did the universe begin in an unstable low-entropy state? Let us first observe that really we are referring not to the "whole" universe (with its gravitational field) but only to the matterradiation subsystem of the universe.

In the no-time version of the introduction we have postulated $\mathrm{H}_{1}$ and $\mathrm{H}_{2}$. Using these hypotheses we have reconstructed time and demonstrated, in the sections above, that the universe expansion creates, in its matterradiation subsystem, an entropy gap $\Delta S$ that takes it out of equilibrium, not only at $t=0$, but in a long period of its history, since the actual entropy is $S_{a c t}<S_{\max }$. We have also demonstrated that the matter-radiation subsystem of the universe evolves to a final state of thermic equilibrium since $\Delta S \rightarrow 0$, when $t \rightarrow \infty$ (cf. eq. ( 75)) ). So the answer to the only problem left is essentially hypothesis $\mathrm{H}_{2}$. Certainly, someone will think that we have solved a problem by postulating an axiom, and this is not a very exiting result. But if the axiom yields the solutions of many problems, and this is the case of $\mathrm{H}_{2}$, the axiom must be welcome. After all, this is the role of axioms.

v.- Finally we can ask ourselves if, in the perspective of the branch system idea, $\mathrm{H}_{2}$ is a natural hypothesis. $\mathrm{H}_{2}$ says that $\rho \in \Phi_{-} \in \mathcal{S} \otimes \mathcal{S}$. So first it is postulated, that $\rho \in \mathcal{S} \otimes \mathcal{S}$, and therefore $\rho$ is a smooth function, with infinite derivatives, and well behaved in the infinities of the configuration space. This part of $\mathrm{H}_{2}$ seems quite natural. Certainly much more natural than the two other alternative possibilities:

a.- $\rho \in \mathcal{L}$, in which case $\rho$ can be, e. g., a square integrable function of $\mathcal{H} \otimes \mathcal{H}$, where in a set of points, the function can take non continuous an arbitrary values. What is the physical meaning of this discontinuity?

b.- $\rho \in \mathcal{S}^{\times} \otimes \mathcal{S}^{\times}$, namely a distribution, e. g. a delta function, certainly a quite unnatural state.

So the first part of $\mathrm{H}_{2}$ is natural. The second part is to ask why $\rho$ would be endowed with a natural asymmetry, the one of $\Phi_{-}$. Is it too much to ask? Let us study this question according to the branch system idea and our formalism. There will be no branch system only if the universe (and now we are referring to the whole universe with the gravitational field included) would begin in an equilibrium state $\rho_{*}$, since in this case it will always remain in equilibrium. Now, from eqs.(64), (65), and (66) we see that, in this case $\rho_{*} \in \Phi_{-}^{\times}$, so $\rho_{*}$ would be a distribution [56], something like a delta function, 
and we have just considered this choice as unnatural. On the contrary $\rho \in \Phi_{-}$ is a much more natural state. Any state of $\Phi_{-}$will produce a branch system, since any state of $\Phi_{-}$yields eq. (65). So we can, at least, conclude that $\mathrm{H}_{2}$ is the requirement that the state of the universe id a natural and an asymmetric one. $\mathrm{H}_{2}$ is also intimately related with the branch system idea and in consequence it is also related with the fact that really our universe is a branch system. $\mathrm{H}_{2}$ is just the transcription of these physical facts.

\section{Coordination of the arrows of time.}

In this section we will only consider the coordination of the arrows of time related with our model, namely:

- The branch arrow of time (BAT), the arrow that goes from the unstable initial state of every process of the universe branch system to its equilibrium final state. As we have seen in the last section this arrow is a direct consequence of the asymmetry introduced by $\mathrm{H}_{2}$.

- The thermodynamic arrow of time (TAT), that points to the direction of the growing of the universe entropy $S$.

- The cosmological arrow of time (CAT), that points to the direction of the growing of the universe scale $a$.

Of course, all these arrows are related with time and therefore they must only be considered in the classical (or semiclassical) period where the time $\eta$, given by eq. (29), is well defined. In the timeless quantum period we only have the asymmetry defined by $\mathrm{H}_{2}$.

Then: eq. (78), for non-expanding or contacting subsystems within the universe, which is a consequence of $\mathrm{H}_{2}$, shows that BAT=TAT, and that $t$, or more generally $\eta$, grows in the same direction than $S$.

The relation between $\mathrm{BAT}(=\mathrm{TAT})$ and $\mathrm{CAT}$ is given by the \pm sign in eqs. (30) or (31). Then these two arrows of time, a priori, are not coordinated in our model. But in the classical period we have just one classical universe and therefore the \pm sign and the constant $C$ are fixed, so in the classical period we have just one sign: either + or - (see App. 12). Therefore, once the sign is fixed, a clear relation appears between BAT and CAT: 
- If the model is an expanding one (and we choose the sign + ) we will have $\mathrm{BAT}=\mathrm{CAT}$, at least in the final evolution where eq. (31) is valid (if we make the unusual choice of the - sign we are just changing the conventional direction of time $\eta$, with no physical consequences).

- If the model is an expanding-contracting one (and we choose the sing $+)$ we will have BAT $=\mathrm{CAT}$ in the expanding period and $\mathrm{BAT} \neq \mathrm{CAT}$ in the contracting period. BAT $=\mathrm{CAT}$ is, in fact, the definition of the expanding period and $\mathrm{BAT} \neq \mathrm{CAT}$ is the definition of the contracting one. But BAT=TAT does not change when we go from the expanding to the contracting period or vice versa, since the choice of $\Phi_{-}\left(\right.$or $\left.\Phi_{+}\right)$ is made once and for all.

So the study of the correlation of the arrows of time is completed, and almost trivial, because we have $\mathrm{H}_{2}$ that defines BAT. (See also [57])

\section{Other results.}

The main results related with quantum cosmology are stated in the above sections. But we must comment that using the present formalism all the relevant results of irreversible statistical mechanics can also be obtained, e. g. all the results of the book [58], as it is proved in ref. [34], because the main $\Pi$ projector of the quoted book can be defined using Gel'fand triplets. Also, in some simple cases, we can go from the quantum models to the classical ones [59], where we find the same philosophy, in classical cases. Chaotic models like Baker's transformation and Renyi's maps, are also treated with the same method, with good results 60. Other interesting results are contained in papers [16], 35], [36], 339], and [57]. So what we have explained is just the

quantum cosmological chapter of a general method to deal with irreversible processes.

\section{Conclusions.}

Let us summarize our main conclusions.

i.- All our scheme is based in the existence of a physically admissible state superspace $\Phi_{-}$and of a physically forbidden state superspace $\Phi_{+}$. Thus, the 
time inversion that goes from $\Phi_{-}$to $\Phi_{+}$is also forbidden. Namely, no Maxwell demon can change the direction of all the velocities of the universe. This is, of course a practically impossible task. Is it also theoretically impossible? In fact it is, even if Maxwell demon would change all these directions (while we are sleeping) we will not notice the change (when we wake up), since all arrows of time would be changed and we would not have any extra arrow to verify the change. Thus this global-demon task is conceptually impossible.

ii.- What we have presented is not a mathematical theorem, but a model that can be generalized in many ways. These more general models will have a similar behavior, than the present one, if two essential features are present: the existence, at the quantum gravity level, of a geometry of maximal relative probability which allows us to construct "out" states for the fields, and a Smatrix with infinite complex poles. The first requirement seems natural for any realistic model of universe. On the other hand we have restricted the class of possible potentials in order to be able to prove that the corresponding Smatrix has infinite complex poles. But several QC potentials do not belong to this class, because they have a bad behavior at infinity. Nevertheless, usually, they also have an infinite set of poles, as it can be proved case by case [59]. So the two basic features seem usual enough to consider that our model is a good sample of the general behavior of the universe. Then we can say that:

iii.- If we introduce an adequate regular state space (or an adequate topology) it seems that all the known results of statistical irreversible physics can be reobtained. It must be emphasized that we are not adding a new object to the theory, since a regular state space (or the corresponding topology) must be defined anyhow. We are just choosing the most convenient one. Let us repeat the general relativity example: The space-time has a metric, we can choose a flat space-time metric or a curved one. In the second case we explain gravity in the best possible way. We add nothing, we just choose the best mathematical structure. The same thing happens in the present case. If we choose the usual regular state space $\mathcal{S}$ we are forced to make a coarse graining (and there is nothing experimentally wrong with coarse graining, as there in nothing experimentally wrong with post-newtonian theories, but both are "non economical" formalisms). If we choose the new regular state space $\phi_{-}$we make two steps in one, so we have a conceptual advantage.

iv.- Precisely, because the new formalism is conceptually clearer we can see that time-asymmetry is just a kind of spontaneous symmetry breaking. 
v.- Most probably the old and new formalism will always yield the same physical results, because they are both based in the same physical base: the limited amount of information must be somehow worked out to obtain a complete theory. Therefore, most likely, they are as experimentally equivalent as general relativity and post-newtonian gravity with an infinite number of terms.

So, even if we have not found any new or spectacular result, we think that the introduced formalism presents a quite coherent picture of the real time-asymmetric universe and shows us how time-asymmetry forces us to choose a Gel'fand triplet as the mathematical structure of the theory.

\section{Appendix. Time-asymmetry of the regu- lar states superspace.}

It is a common lore among physicists that, since the fundamental evolution equations are time-symmetric and the universe is time asymmetric, therefore the state of the universe must be the responsible for time asymmetry. Namely time-asymmetry is created as in the case of spontaneous symmetry breaking. The problem is to mathematically precise this idea. That is what we have done in this paper, by showing that the asymmetry can be hidden in the choice of an asymmetric regular state superspace. In fact, beginning with the one symmetric regular state space $\mathcal{S}$ of the primitive theory, we have introduced the asymmetry related with the lower and upper halfplanes, of the complex plane, and we have defined asymmetric regular state spaces $\phi_{-}$and $\phi_{+}$, and we have chosen one of them, say $\phi_{-}$, creating a symmetry breaking.

But, since this is perhaps the most important point, we will try to explain this fact in the most didactical way. First let us consider that $a \geq 0$, and let us draw the potential $V(\eta)=V[a(\eta)]$ in the right side of the horizontal $\eta$ axis. Let us take $C$ such that $\eta=0$ would correspond to $a=0$ in eq. (31), to make the origin of $a$ to coincide with the one of $\eta$. As, in principle, $\eta$ can be positive or negative we will complete the previous figure with a symmetrical curve in the left side of the vertical axis. Really as we cannot go from negative $\eta$ to positive ones we can add an infinite potential barrier at $\eta=0$. Now we have a more or less typical potential figure for a spontaneous 
symmetry breaking. In our case the minima are unimportant (even if, most likely, the equilibrium states will correspond to the minima in almost every model) but since we have an infinite potential barrier at $\eta=0$ it is clear that the universe can either be in the right or the left side of the vertical axis. Choose either one or the other possibility. Then, you have chosen the + or the - sign in eqs. (30) or (31) (since we have chosen $a=0$ when $\eta=0$ and it is always $a \geq 0)$. Let us consider, for a moment the expanding case: if you choose + then BAT $=\mathrm{CAT}$ and you have chosen the convention of all the formulae of this paper, namely you have chosen a $\mathrm{H}_{2}$ with a $\Phi_{-}$space as in this paper. If you choose the - sign, you just change the convention used in this paper, instead of $\eta$ you will have $-\eta$ everywhere and in $\mathrm{H}_{2}$ you will have $\Phi_{+}$. Of course, physically nothing is changed since you have just changed a convention. In the expanding-contracting case we can repeat the reasoning for the expanding phase only and everything turns out to be the same.

So we are clearly in a case of a symmetry breaking, produced by the state space and not by the equations, where we have conventionally chosen between two conventional different possibilities, either the right side of the figure or the left side, namely between $\Phi_{-}$and $\Phi_{+}$. After this choice the time-asymmetry appears but, of course, the choice itself is irrelevant, as in every spontaneous symmetry breaking. So really there are two \pm choices in the theory: the one that corresponds to the choice between $\Phi_{-}$and $\Phi_{+}$and the one in eqs. (30) and (31). We will explain more about the second choice in the next appendix.

\section{Appendix. The asymmetry of $\mathrm{Q}$ space.}

Why do we have + and not - in eqs. (30) or (31) ? Perhaps it is interesting to relate the present formalisms with the final speculations of the second paper [9]:

If $\Phi_{-}$is chosen (namely the first \pm choice is made)and $C>0$ : Are the + and -, of eq. (31) conventionally identical? Is the choice between them just conventional or is there something substantially different between them? Of course the difference between + and - is just impossible to find in the quantum gravity timeless period. But the arena of time asymmetry is the classical period where the \pm sign and the constant $C$ are fixed (and as in this

case we have taken $C>0$, as we have said). Can we find a difference between 
+ and - in this period? Our strategy is to maintain the symmetry the WDW equation and create the time-asymmetry using the states. In doing so we can use all the features of the states and the configuration space $Q$. And it turns out that $Q$ is not so symmetric since it has boundaries [9] that gives $Q$ some peculiar structure. Precisely $a \geq 0$ in our very simple model. So once the choice of the space of state is made, using all the well known philosophy of symmetry breaking, as explained in section Appendix 11, + and - become obviously different in expanding models since:

- With the + we would have equilibrium towards $a \rightarrow \infty$, which is possible.

- With the - we would have equilibrium towards $a \rightarrow-\infty$, which is impossible since $a \geq 0$.

So we must choose the + . If we would have begun choosing $\Phi_{+}$(and $C<0)$ we will be forced to choose the - sign. So the choice of the regular state space implies the choice of a sign. In expanding-contacting models things are more difficult since we do not have either $a \rightarrow \infty$ or $a \rightarrow-\infty$, so theoretically we never reach the equilibrium state. In these models we must change the notion of equilibrium, and consider that this state is obtained in long but finite times. Then, we must consider only the expanding phase and repeat the reasoning of expanding models.

Therefore, as it was already anticipated in ref. [9] the boundary structure or $Q$ creates a difference, and allows us to choose the right sign.

\section{Acknowledgments.}

I would like to warmly thank R. Aquilano, J. Barbour, and M. Gadella for many stimulating discussions and to several colleagues, for a great number of interesting questions, that I try to answer in the references.

This work was partially supported by grants: CI1*-CT94-0004 of the European Community, PID-0150 of CONICET (National Research Council of Argentina), EX-198 of the Buenos Aires University, and 12217/1 of Fundación Antorchas and the British Council. 


\section{References}

[1] Castagnino M., Jour. Mat. Phys., 12, 2203, 1971.

[2] De Witt B., Phys Rev. 160, 1113, 1967.

[3] Halliwell J., Introductory lectures on quantum gravity, Proc. Jerusalem Winter School on Quantum Gravity and Baby Universes. Piran T. ed., 1990 .

[4] Halliwell J., Perez Mercader J., Zurek W. H. Eds. Physical origin of time asymmetry, Cambridge Univ. Press, Cambridge, 1994.

[5] Hartle J.,Quantum Cosmology, in High energy physics 1958: Proc. Yale Summer School, Bowik M. J., Gursey F. Eds., World Sci. Singapore, 1985.

[6] Hawking S., Page D. N., Nucl. Phys. B 298, 789, 1986.

[7] Castagnino M., Ganghi A., Mazzitelli F. D. Tkachev I. I., Class. Quant. Grav. 10, 2495, 1993.

[8] Kuchar K. Time and interpretation of quantum gravity, Proc. 4th. Canadian Conference on General Relativity and Relativistic Astrophysics, Kunstatter G., et al. eds., World Sci., Singapore, 1992.

[9] Barbour J. B. Class Quant. Grav., 11, 2835, 1994 and 11, 2875, 1994.

[10] In fact, the state of the universe, in this period, is described by a density matrix $\rho\left(q, q^{\prime}\right)$ where $q, q^{\prime} \in Q$. Among all the configuration variables $q$ we may choose a particular one, as the hand of our clock, let us say the radius of the universe $a$. Then $\rho\left(q, q^{\prime}\right)=\rho\left(a, x, a, x^{\prime}\right)$, where $x$ symbolizes the rest of the variables. But to use, $a$ as the hand of our clock, it is necessary to have decoherence for $a \neq a^{\prime}$, e. g. $\rho\left(a, x, a^{\prime}, x^{\prime}\right)=0$ if $a \neq a^{\prime}$ or $\rho\left(a, x, a^{\prime}, x^{\prime}\right) \sim \delta\left(a-a^{\prime}\right) \rho\left(a, x, x^{\prime}\right)$. Namely, at least one variable, e. g. $a$, must have classical properties, if not we will be forced to make a theory with two "times" $a$ and $a^{\prime}$. But if we have just one $a$ we are not more in full QC, but in a semiclassical regime, as those of section 4, where in fact, we will find decoherence of the radius $a$ [23]. We conclude that, even if some generalization of time may be defined in full quantum 
gravity period, the usual time, with its known properties, cannot exist in such regime.

[11] We have defined the time, for the semiclassical phase of the universe (even with back-reaction) in the following papers:

Castagnino M., Phys Rev D. 39, 2216, 1989.

Castagnino M., Mazzitelli F. D., Phys Rev. D. 42, 482, 1990.

Castagnino M., Mazzitelli F. D., Int. Jour. of Theo Phys. 34, 47, 1989.

Castagnino M. Lombardo F., Phys. Rev. D. 48,1722, 1993.

But it was impossible for us to extend this definition to the quantum period.

[12] Isham C., Canonical quantum gravity and the problem of time, in Recent Problems in Mathematical Physics, Salamanca, 1992.

[13] Bogolubov N. N., Logunov A. A., Todorov I. T., Introduction to axiomatic quantum field theory, Benjamin, London, 1975.

[14] If only spaces $\Phi_{-}$and $\Phi_{-}^{\times}$were defined, for every state $\rho \in \Phi_{-}$and every observable $A \in \Phi_{-}^{\times}$(cf. eq.17) we can compute the "mean value":

$$
<A>_{\rho}=A[\rho]
$$

This number is well defined by the linear operator $A$ acting on the state $\rho$. Really this is the maximal answer QC can give: a typical "mean value" for every observable and for every quantum state of the universe, since the ensemble of QC has just one system: the universe. Therefore the" mean value" is not a proper mean value but just a "typical" one.

If we want to know the probability $p(q)$ to find the universe at a certain $q$ and if $Q$ is the position operator:

$$
Q=\int q|q><q| d q
$$

and $\rho$ is a pure state $\rho=|\varphi><\varphi|$, we need to define a norm and the inner product of $\mathcal{H}$ to compute:

$$
p(q)=|<\varphi| q>\left.\right|^{2}
$$


But we know that the notion of probability in an ensemble of just one element in not a reasonable one. So really it is more logical to work just with the couple $\Phi_{-}, \Phi_{-}^{\times}$, and with the typical values $A[\rho]$ than to use the notion of probability. Nevertheless in this paper we will use the more familiar "probabilistic approach" of QC, where we need a norm like 2. We will study the "typical approach" of QC, where the norm is superfluous, in a forthcoming paper.

[15] If $\rho=|\Psi><\Psi|$ is a pure state these equations coincide with WDWequation (11).

[16] Parravicini G., Gorini V., Sudarshan E. C. G., J. Math. Phys. 21, 2208, 1980.

[17] In every reasonable model of the universe it seems to exist a final equilibrium state that acts like an attractor for every initial state of the theory. See e.g.:

Hosotani Y., Phys Rev. D. 32, 1949, 1985.

Demianski, et. al. Phys. Rev. D., 44, 3136, 1992.

[18] As we will see in the model of section 2, we must restore variable $a$ in $\omega$, out $>$, which is really a function of $\eta=\eta(a)$, and then multiply it by an adequate prefactor, to get a solution of WDW equation. With these modifications $\mid \omega$, out $>$ becomes a vector of superspace.

[19] We do not know which are the necessary and sufficient conditions in order to be sure that this structure would exist. Any how there is an obvious necessary condition: the superspace must be time oriented, namely two subsuperspaces $\Phi_{-}$and $\Phi_{+}$must be found such that $K: \Phi_{-} \rightarrow \Phi_{+} \neq \Phi_{-}$, being $K$ the Wigner operator (namely the complex conjugation).

[20] As $\left(H_{-}^{2}\right)^{*}=H_{+}^{2}$ then if $\Psi(\omega)=<\omega$, out $\mid \Psi>\in H_{-}^{2}$ we have $\Psi^{*}(\omega)=<$ $\omega$, out $\mid \Psi>^{*} \in H_{+}^{2}$. Then we can foresee that the substitution of $\Phi_{-}$by $\Phi_{+}$will become the time inversion when time will be defined, namely $K$, the Wigner operator, will become this inversion.

[21] Of course, we will have such kind of continuous spectrum, only if the spatial geometry of the universe is open. In closed models we can only 
suppose that the discrete eigenvalues are so closed that they can be considered as a continuous spectrum in some approximation. This fact, and the problems that we will face with an expanding-contracting universe, shows that our formalism is much better adapted to open geometries

[22] Paz J. P., Sinha S., Phys. Rev. D. 44, 1089, 1991.

[23] Castagnino M., Lombardo F., Gen. Rel. and Grav, 28, 263, 1996.

Castagnino M., Gunzig E., Lombardo F., Gen. Rel. and Grav. 27, 257, 1995 ,

[24] Following D. Bohm we can also say that WDW equation is exactly equivalent to the system:

$$
\begin{aligned}
& {\left[S^{\prime}(a)\right]^{2}=2 V(a)+\frac{i}{2 M^{2}} S^{\prime \prime}(a)} \\
& i S^{\prime}(a) \frac{\partial}{\partial a} \chi=h \chi-\frac{1}{2 M^{2}} \frac{\partial^{2}}{\partial a^{2}} \chi
\end{aligned}
$$

where the $M^{-2}$ order corrections can be considered as a gravitational correction to the Hamilton-Jacobi equation and as a quantum potential that must be added to the classical potential of hamiltonian $h$. Theoretically this system can be exactly solved. See:

Bohm D., Hiley B. J. The undivided universe, Routledge London, 1993.

[25] Castagnino M., Gen. Rel. and Grav., 15, 1149, 1983.

Castagnino M., Mazzitelli F. D., Phys. Rev. D, 31, 742, 1985.

[26] Birrell N. D., Davies P. C. W., Quantum fields in curved space, Cambridge Univ. Press, Cambridge, 1982.

[27] Audrecht J., J- Phys. A: Math. Gen. 12, 1189, 1979.

[28] Nussenzveig H. M. Causality and dispersion relations, Academic Press, New York, 1972.

[29] Birrell N. B., Taylor J. G., J. Math Phys., 21, 1740, 1980. 
[30] Precisely and repeating what we have anticipated in $\|18\|, \mid \chi>\in \phi_{-}^{\text {out }}$ will be a function of $\eta$ and a functional of field $\varphi$. But $a=a(\eta)$ so $\chi=\chi(a, \varphi)$ is the function of eq. (27), that multiplied by the prefactor $\exp [i M S(a)]$ is $\Psi(a, \varphi)$, a solution of the WDW equation. So it is $\Psi(a, \varphi)$ the function that really belongs to $\phi_{-}$. This fact proves that the asymmetry exists either in the spaces of $\chi$ or $\Psi$ functions and therefore in the full quantum superspace.

[31] The main difference of this curved space-time formalism, with the flat space usual one, is that in the former case we have two unequivalent vacua; $\mid 0$, in $>$ and $\mid 0$, out $>$, while in the latter one we have just one: $\mid 0>$, such that $K|0>=| 0>$, where $K$ is the Wigner time-reversal operator. In the curved space time case $K \mid 0$, in $>\neq \mid 0$, out $>$ and therefore we have two equations:

$$
\begin{gathered}
K: \phi_{-}^{\text {out }} \rightarrow \phi_{+}^{\text {out }} \neq \phi_{-}^{\text {out }} \\
K: \phi_{+}^{\text {in }} \rightarrow \phi_{-}^{\text {in }} \neq \phi_{+}^{\text {in }}
\end{gathered}
$$

In the usual case the "in", "out" superscript would be absent and therefore, there would only be one equation. These differences, with the usual case, must be taken into account but they are not very important.

[32] Castagnino M., Verbeure A.,Weder R., Nuovo Cimento, 26, 396, 1975.

[33] Eventually the location of the poles can be such that it turns out to be impossible to find the curve $\Gamma$. Then we can use a set of curves $\left\{\Gamma_{n}\right\}$, such that $\Gamma_{n}$ goes below the poles $\Omega_{1}, \Omega_{2}, \ldots \Omega_{n}$, and take $n \rightarrow \infty$ at the end of calculations

[34] Castagnino M., Gaioli F., Gunzig E., Cosmological features of time asymmetry, Found. Cos. Phys., in press, !995.

[35] Bohm A., Quantum mechanics: foundations and applications, Springer Verlag, Berlin, 1986.

Bohm A., Gadella M., Maynland B. G., Am. J. Phys., 57, 1103, 1989.

Bohm A., Gadella M., Dirac kets, Gamov vectors, and Gel'fand triplets, Springer Verlag, Berlin 1989. 
[36] Sudarshan E. C. G., Chiu C. B., Gorini V., Phys. Rev., D 18, 2914, 1978. Eq. (81) can be obtained if we eliminate the short time effect (Zeno effect) and the long time effect (Khalfin effect). These modifications explain why we obtain a growing entropy instead of a constant one.

[37] Nakanishi N., Prog. Theor. Phys. 19, 607, 1958.

[38] Castagnino M., Domenech G., Levinas M. L., Umerez N., The doublet representation of non-Hilbert eigenstates of the hamiltonian. J. Math. Phys. in press 1995.

[39] Antoniou I., Prigogine I., Physica A 192, 443, 1993.

[40] Castagnino M., Laura R., On a minimal irreversible quantum mechanics, submitted to Phys. Rev. A, 1995.

[41] In a practical complete case microscopic motions would always remain. These microscopic motions are, in our model, those of the field, namely those that correspond to the integral in the spectral decomposition. In practice they have a maximum (very small) length, since the integral, in the spectral decomposition really does not begin with zero , but with a finite value. If we would introduce a coarse-graining these microscopic motions would be hidden and the equilibrium would be the usual stationary equilibrium state $\rho_{*}=$ const. But in our formalism there is no need of a coarse-graining to hide the microscopic motions. Really we have only a mathematical graining (the choice of the right mathematical structure) to produce time asymmetry. The macroscopic energy, we had at the beginning, goes into microscopic oscillations, in order to fulfill the law of energy conservation. Also if we multiplied eq.(65) by a smooth distribution $\sigma$, the microscopic oscillation would be smeared, $\left(\rho_{*}(\eta) \mid \sigma\right)$ becomes a constant and we have a typical equilibrium weak limit [42]: $\lim _{\eta \rightarrow \infty}(\rho(\eta) \mid \sigma)=\left(\rho_{*}(\eta) \mid\right.$

[42] Mackey M. C., Time's arrow: the origin of thermodynamic behavior, Springer Verlag, Berlin, 1992.

Lasota A., Mackey M. C., Probabilistic properties of thermodynamic behavior, Cambridge Univ. Press, Cambridge, 1985.

Mackey M. C., Rev. Mod. Phys., 61, 981, 1989. 
[43] Castagnino M., Gadella M., Gaioli F. Laura R., Gamov vectors and time asymmetry, in preparation, 1995.

[44] As in this equation distributions are multiplied, some care must be taken in order to convince ourselves that what we are doing is mathematically correct. E. g. the distributions can be transformed in ordinary density matrices by a $\Lambda$ transformation [34]. This transformation maintains the dumping factors, so the results obtained remain valid, but the distributions become ordinary matrices, that can be multiplied. In this way the r.h.s. of eq. (74) becomes well defined. There are also more refined mathematical ways, to reach to the desired result, as the one of ref. [40], part III, and those based on the theory of locally convex spaces (in preparation).

[45] Calzetta E., Castagnino M., Scoccimarro R., Phys. Rev. D. 45, 2806, 1992.

[46] Davies P. C. W. Stirring up trouble, in Physical Origin of Time Asymmetry, Halliwell J. J. et al. eds., Cambridge Univ. Press, Cambridge, 1994.

[47] Aquilano R., Castagnino M., The entropy gap and time asymmetry, Mod. Phys Let. A, in press, 1996, The lack of thermic equilibrium as a consequence of the universe expansion, Astr. and Space Sci., in press, 1996.

[48] Tolman R. C., Relativity, thermodynamics, and cosmology, Dover Pub., New York, 1987.

Misner C. W., Thorne K. S., Wheeler J. A., Gravitation, Freeman and Co., San Francisco, 1970.

[49] Peebles P. J. E., Principles of physical cosmology, Princeton Univ. Press, Princeton, 1993.

[50] Landau L. D., Lifshitz E. M., Statistical Physics, Pergamon Press, Oxford, 1958.

[51] Jones C., Forman W., Cluster and supercluster, in NATO ASI, vol. 366, Fabian A. C. ed., Dordrecht, Kluwe, 49, (JF), 1992. 
[52] Reeves H., The growth of complexity in an expanding universe, in The Anthropic Principle, Bertola F., Curi U. eds., Cambridge Univ. Press, Cambridge 1993.

[53] Dicus D. A., Letaw J. R., Teplitz D. C., Teplitz V. L., Effect of proton decay on the cosmological future,.Astrophysical Jour., 252, 1, 1982.

[54] Reichenbach H., The direction of time, University of California Press, 1956.

[55] Zwanzig R. W., Chem Phys., 33, 1338, 1960.

Zwanzig R. W., Statistical mechanics of irreversibility, in Quantum Statistical Mechanics, Meijer P. ed., Gordon and Breach, New York, 1966.

Zurek W. H., Physics Today, 44, (10), 36, 1991.

[56] This fact is evident if we consider a Baker's transformation [42]: Let us consider a distribution function with compact support, and such that it belongs to $\mathcal{L}=L^{2}([0,1] \times[0,1])$. In the far future this support becomes a set of horizontal strips, and in the limit a set of horizontal straight lines, such that it is dense in $[0,1] \times[0,1]$. No function of $\mathcal{L}$ has such a support and therefore the equilibrium state does not belong to $\mathcal{L}$.

[57] The quantum arrow of time (QAT), that goes from preparation to measurement, and coincides with the collapse arrow of time, can also be considered, as in papers:

Bohm A., Phys. Rev. A, 51, 1758, 1995.

Bohm A., Loewe M, Maxson S., Microphysical irreversibility in quantum mechanics, Reports on Theoretical Phys., in press, 1995.

Antoniou I, Bohm A., Kielanowski P., J. Math. Phys., 36, 1, 1995.

It is not difficult to see that this arrow also coincides with BAT=TAT, since the measurement process is an irreversible decoherence process which is also contained in (Reichenbach) branch system and, therefore, the measurement arrow (QAT) must coincide with BAT.

[58] Balescu R., Equilibrium and non-equilibrium statistical mechanics, J. Wiley \& Sons, New York, 1963. 
[59] Castagnino M., Diener R., Lara L., Puccini G., Instability and timeasymmetry: the case of the upside down simple harmonic oscillator, submitted to An. of Phys (N. Y.), 1995.

[60] Antoniou I., Tasaki S., Physica A, 190, 303, 1991.

Antoniou I., Tasaki S., Int. J. Quant. Chem., 46, 427, 1993 